\title{
QSe
}

José Luis Atristain*

Pilar Randolph**

\section{LA LETRA PEQUEÑA DE LA EXPORTACIÓN DE ALIMENTOS A CANADÁ}

Aunque Canadá es un país abierto al comercio internacional, sin embargo, la sobrerregulación de sus procesos de importación y trabas no arancelarias, particularmente en el sector alimentario, hacen muy complicada la entrada de nuevos suministradores. El objetivo de este artículo es mencionar los casos más sobresalientes en los sectores clave como lácteos, cárnicos y bebidas alcohólicas y prevenir al exportador español de las limitaciones y trabas que seguramente encontrará de cara a abrirse paso en este mercado. La metodología seguida resume la experiencia acumulada de la Oficina Económica y Comercial y el análisis de la multitud de requerimientos, procesos y restricciones que existen a la importación de productos alimenticios (incluidos los vinos) en Canadá, así como la consulta de las fuentes oficiales canadienses.

Palabras clave: barreras no arancelarias, sistemas de control, alimentación, lácteos, liquor boards. Clasificación JEL: F13, L66.

\section{Introducción y advertencia}

La historia del comercio entre España y Canadá nos indica que esta relación bilateral se ha desarrollado siempre supeditada al contexto de otras prioridades comerciales de ambos países. Tanto España como Canadá, países integrados en distintas áreas económicas y ubicados en distintos continentes, pocas veces se miran de frente, y sus exportadores en general tienen poca o nula conciencia del potencial de sus productos en el mercado alterno. Los

\footnotetext{
* Analista de Mercado de la Oficina Económica y Comercial de España en Toronto.

** Jefa del Departamento de Alimentación y Bebidas de la Oficina Económica y Comercial de España en Toronto.

Versión de septiembre de 2021.

https://doi.org/10.32796/bice.2021.3140.7290
}

exportadores españoles, bastante ocupados en aprovechar un amplio y creciente mercado natural de 27 países de la Unión Europea, difícilmente vuelven su atención hacia el mercado canadiense, mientras que Canadá, inmersa en su ámbito norteamericano y manteniendo la mayor relación de intercambio bilateral del mundo con la primera potencia industrial y económica del planeta (a través de 7.000 kilómetros de frontera con Estados Unidos) solo mira a Europa cuando intenta colocar excedentes de sus exportaciones de materias primas.

La anterior situación ha creado la aseveración, muy próxima a la realidad y frecuentemente mencionada en los escasos encuentros bilaterales de alto nivel, de que «España y Canadá comercian en un año el valor en $\triangleright$ 
mercancía que USA y Canadá se intercambian en un día». Tal aseveración, aunque estadísticamente correcta, no valora todos los ámbitos de la relación bilateral que incluye las grandes inversiones en ambos sentidos y tampoco pone de manifiesto el proceso de diversificación que se ha dado recientemente, sobre todo, en las exportaciones españolas a Canadá.

Aun así, la relación comercial y de inversión bilateral entre España y Canadá crece firme, aunque de manera muy pausada, y atribuimos esta conducta al hecho de que estos mercados, tanto el canadiense como el español, a partir de la globalización, paradójicamente, han debido acogerse a sus mercados naturales.

Aunque es un tanto prematuro para opinar cabalmente, resulta importante también señalar que ni siquiera la implementación del Acuerdo Integral Económico y de Comercio entre la Unión Europea y Canadá (CETA), que entró en vigor el 21 de septiembre de 2017, ha podido dar el vuelco cuantitativo esperado a la relación comercial entre España y Canadá.

Las exportaciones sectoriales españolas a Canadá, mucho más diversificadas que en sentido opuesto, sufren casi siempre de un desarrollo muy errático. Lo anterior se debe a su característica de abordarse siempre como un mercado adyacente del mercado americano.

La fragmentación geográfica de Canadá y su reducida dimensión, en comparación con el mercado de su vecino del sur, no favorecen al país como mercado objetivo importante en el horizonte de la mayoría de los exportadores españoles. La experiencia de nuestras Oficinas Económicas y Comerciales indica que solo aquellas empresas españolas que abordan el mercado canadiense no como un apéndice del mercado americano, sino como una entidad distinta e integral, han podido coronar sus esfuerzos con éxitos comerciales consolidados.
Lo anterior nos lleva al meollo del presente artículo, que pretende enumerar de manera extensa pero nunca completa, ni tampoco en detalle, las principales barreras no arancelarias a las que el exportador español (en materia de productos alimenticios) se enfrenta cotidianamente y que son raramente advertidas cuando se aborda por primera vez el mercado de productos agroalimenticios de Canadá.

Estos comentarios no son, ni de lejos, una denuncia de trabas al comercio establecidas por parte del Gobierno o entidades privadas canadienses en contra de los exportadores españoles interesados en el mercado canadiense. La multitud de normas, reglamentos y demás disposiciones que afectan a la importación de productos agroalimentarios es de aplicación general a cualquier mercancía procedente de cualquier origen y, sobre todo, se trata de las mismas normas que rigen para los productos canadienses y para los productores y fabricantes locales.

En múltiples ocasiones hemos sido testigos presenciales de que la aplicación de normas y restricciones tanto sanitarias como de carácter de contingencia estratégica a las mercancías españolas es totalmente transparente, equitativa y apegada a la norma escrita establecida por parte de las autoridades canadienses.

Sin embargo, la fama de Canadá como nación abierta al libre comercio y al libre intercambio de productos, a la luz de muchas de estas restricciones y cortapisas gubernamentales en muchos sectores del comercio internacional, sufre mucho y podría considerarse mejor como un país abierto al comercio, sí, pero altamente protegido en bastantes sectores importantes y sobrerregulado.

No discutimos aquí la justificación o no de tales políticas así establecidas por los Gobiernos canadienses a través de los años, sino $\triangleright$ 
simplemente nos proponemos enunciar varios ejemplos de tales restricciones y sus subsecuentes cortapisas al libre comercio con el único objetivo de que los exportadores españoles interesados en Canadá tengan una referencia cabal de las condiciones de acceso no evidentes a primera vista y que condicionan severamente el acceso al mercado canadiense y su desarrollo en el futuro.

El lector encontrará conclusiones individuales en los varios incisos que componen este trabajo, todas conducentes a advertir que la sobrerregulación del mercado canadiense es un aspecto muy importante a determinar en el momento de establecer la viabilidad de cualquier producto alimenticio español de cara a introducirse en el mercado canadiense.

\section{Sistemas de control de la oferta de productos agrícolas en Canadá (supply management in Canada)}

Históricamente, Canadá es un país que nace como respuesta a una confrontación bélica entre el Imperio británico y los primeros anhelos expansionistas de los Estados Unidos de América. Su historia es la opuesta a la declaración de independencia de Washington; los territorios que permanecieron fieles a la Corona británica empiezan a agruparse como entidades francesas o inglesas como un reflejo de las tensiones entre las potencias europeas durante el siglo XIX.

Después de la invasión americana, durante la Guerra de 1812 entre Inglaterra y los Estados Unidos de América, empieza en los territorios que hoy son Canadá un decidido proceso de constitución de provincias e intentos de formar una confederación en los enormes territorios que la Corona inglesa poseía en Norteamérica.
Estas provincias buscaban protección mediante la unión y defensa militar inglesa ante la evidente e inminente expansión territorial americana que sucedía a lo largo y ancho del continente.

En 1867, con la consolidación de un Parlamento y un Gobierno arropados por la Corona inglesa, se da paso a la creación de una entidad confederada a la cual se van añadiendo provincias nuevas, que con el paso del tiempo se constituyen en la monarquía parlamentaria canadiense.

Con la repatriación de la Constitución política del Parlamento inglés a Ottawa en 1982 y sus primeras enmiendas concluye el proceso de consolidación del Estado federal canadiense. Sin embargo, en la nueva constitución política se mantienen las competencias importantes de las antiguas entidades provinciales federadas, las mismas que se mantienen hasta la fecha. Este enorme poder provincial se garantiza gracias a los pactos de distribución equilibrada de jurisdicción y competencias con el Gobierno federal.

Siendo Canadá desde principios del siglo xx una potencia agrícola, particularmente por su producción de cereales y lácteos (gracias a su vasta extensión territorial de cerca de $10 \mathrm{mi}$ llones de kilómetros cuadrados), pero sobre todo debido a su vecindad con un mercado creciente, diversificado y en expansión como el de Estados Unidos, muy pronto permitió que los granjeros canadienses desarrollaran una enorme expansión de su producción agrícola.

Debido también a la enorme extensión territorial canadiense (con cinco husos horarios distintos), a partir del inicio del siglo xx empieza a darse un acelerado proceso de inmigración proveniente de países europeos, predominantemente del Reino Unido. Estos nuevos canadienses se integran en actividades de industrias muy básicas como la construcción, $\triangleright$ 
pero la mayoría se dedica a actividades agropecuarias en Ontario y en las denominadas provincias de la pradera canadiense, Manitoba, Saskatchewan y, en la costa pacífica, la Columbia Británica.

El auge económico experimentado en todo el continente norteamericano al final de la Segunda Guerra Mundial permitió la consolidación económica de una clase agraria en Canadá que empieza a demandar tecnologías modernas de producción para afrontar la demanda americana de productos alimenticios. Casi por ósmosis, al norte de la frontera se va desarrollando un importante mercado interno cada vez más boyante que se empieza a concentrar en ciudades como Montreal, Toronto y Vancouver, principalmente, pero también en centros urbanos de menor escala como Calgary, por su potencial petrolero, y Winnipeg y Saskatoon, por su producción ganadera.

Por lo anterior, no es raro que, al inicio de la década de los sesenta, coincidan dos fenómenos en Canadá, uno social y el otro económico, que van a cambiar tanto las formas de producción como la distribución de productos agrícolas en Canadá. Por una parte, la concentración de nuevos inmigrantes en las grandes ciudades canadienses permite el desarrollo de un mercado interno que, debido a la influencia cultural de EE. UU. en Canadá a través de la televisión, unifica hábitos de consumo en ámbitos casi siempre urbanos o suburbanos. Este mercado, casi virgen, es suministrado por productores estadounidenses y atendido por un número reducido de grandes corporaciones canadienses que operan las grandes cadenas de supermercados y que propician una concentración de la demanda de alimentos frescos en muy pocas manos.

Por otra parte, el boom demográfico que surge en el continente después del final de la
Segunda Guerra Mundial plantea un reto tecnológico que nace de la necesidad de suministrar grandes cantidades de alimentos frescos a núcleos de población cada vez más grandes en las ciudades. Esta industrialización de los métodos de producción de alimentos propicia la inversión en el desarrollo de técnicas de preservación industrial de alimentos donde los procesos de la pasteurización y refrigeración de lácteos, el uso de aditivos y químicos estabilizadores y, finalmente, el envase automatizado de productos alimenticios en lata y plástico permiten a las cadenas de supermercados no depender tanto de alimentos frescos de las granjas.

La alta concentración de la demanda alimenticia en pocos grupos de distribución produce una situación de competencia por el suministro, donde las cadenas pagan cada vez menos por los productos frescos de los granjeros. Estos, individualmente, intentan compensar estas pérdidas de negocio adquiriendo equipos de automatización para incrementar su producción y así tener precios más competitivos que sus vecinos. En tales circunstancias, los granjeros canadienses optan por incrementar su producción, particularmente de leche, huevos y sus derivados, creándose así una enorme burbuja de suministro que deprime aún más los precios de sus productos.

Hacia los años cincuenta, esta situación de crisis en el campo se generaliza en varias provincias importantes como Ontario, Quebec y la Columbia Británica, donde un gran número de granjeros empieza a declarase en suspensión de pagos y recurre a los Gobiernos provinciales en busca de ayudas y protección. Los Gobiernos, tanto federal como provinciales, intentan solucionar la crisis mediante la concesión de subvenciones, pero muy pronto se dan cuenta de que la crisis empieza a generalizarse más $\square$ 
allá de su posibilidad de subvención, y la pérdida de empleos y granjas se transforman en motivo de críticas agrias a los partidos en el poder.

Tanto los Gobiernos provinciales como la Administración federal buscan resolver la crisis $y$, en conjunción con organizaciones de productores, diseñan soluciones de emergencia en forma de acuerdos y pactos entre asociaciones agrícolas para evitar las guerras de precios entre los propios granjeros.

Los primeros experimentos se dan mediante cámaras de compensación y fijación de precios de referencia en aquellos sectores que por su población empleada y volumen sectorial resultan más críticos.

Así, en 1966, se crea la denominada Canadian Milk Supply Management. De acuerdo con el Plan Nacional de Mercado de la Leche, establece la Canadian Dairy Commission, una entidad federal de la Corona (Crown Corporation) con facultades para establecer medidas de control sobre la producción, distribución y fijación de precios de referencia para todos los productos lácteos en Canadá, con el fin de controlar el mercado y proteger a los granjeros de las fluctuaciones erráticas de precios y defender al sector productivo de la competencia de la distribución, que se consideró desleal en aquellos momentos.

Rápidamente, los granjeros de otros subsectores de la producción agrícola canadiense decidieron agruparse y seguir el ejemplo de la Canadian Milk Supply Management. Y así, surgieron al final de la década de los setenta organizaciones como Eggs Farmers of Canada (1972), Turkey Farmers of Canada (1974), Chicken Farmers of Canada (1978) y Canadian Hatching Eggs Producers (1986).

Con estas medidas de política pública, el Estado federal canadiense transforma mercancías de primer uso en commodities con precios de referencia, que van a controlar y deformar el mercado interno de alimentos en el país.

Obviamente, si se controla el mercado interno, se obliga al Estado canadiense a controlar la importación de esos productos provenientes de otros países, particularmente del enorme potencial productivo de Estados Unidos, su vecino del sur.

A través de los años ha existido en Canadá una gran polémica sobre la aplicación de estas medidas de protección a los granjeros. Los defensores del control sobre el suministro de productos alimenticios propugnan que tales organismos controlen el mercado de productos básicos que, de otra manera, dejando su comportamiento a las fuerzas brutas del mercado, afectarían al bolsillo del consumidor canadiense. Asimismo, indican que estos sistemas de control de precio permiten al productor de artículos de primera necesidad planificar a futuro, crecer y ser eficientes, al tiempo que protege el empleo en sus granjas.

Los granjeros señalan, además, que estos controles a la producción de alimentos básicos evitan los ajustes cíclicos de crecimiento acelerado y crisis subsecuente, cuyos ejemplos tan frecuentes como desastrosos abundan en los sectores agropecuarios de Estados Unidos.

Subrayan estos defensores del control de precios que este sistema permite mantener en crecimiento y evolución constantes a un sector crítico de la economía para beneficio directo del consumidor canadiense.

Finalmente, y en esta misma línea, bastantes autores afines al sistema de control de precios señalan que si desmantelan estos sistemas, tal y como sugieren algunas instancias internacionales, EE. UU. inundaría el mercado canadiense con sus enormes excedentes de producción, lo que llevaría a la quiebra a miles de granjeros canadienses que mantienen la soberanía alimentaria de este país. 
Sin embargo, existen en Canadá también muchos detractores de estos sistemas. Desde el sector privado hay muchos sectores críticos del sistema de control oficial de suministros que normalmente aluden a la pobre relación costo-beneficio de las operaciones bajo control gubernamental, argumentando que el resultado final son precios más altos de los artículos básicos al consumidor canadiense y un retorno económico menor a los productores debido a la intermediación gubernamental.

Estos sectores críticos argumentan, además, que solo una pequeña parte del alto precio que paga el consumidor final llega al granjero, debido al alto costo burocrático necesario para mantener enormes estructuras de supervisión y administración del sistema de control de suministro. Otras voces mencionan la escasa transparencia y el abuso de subsidios para los productores de algunas provincias «privilegiadas» debido a su alto poder electoral.

Finalmente, pero quizás de manera más fundamental para los efectos de este trabajo, están los argumentos de los críticos del sistema de control sobre el suministro de productos alimenticios, en el sentido del grave daño que la implementación de tales sistemas tiene sobre la imagen de Canadá ante los organismos internacionales de comercio, en cuanto a prácticas que afecten la libertad de comercio.

Baste mencionar el enorme debate suscitado recientemente durante la negociación internacional de la entrada de Canadá en el acuerdo comercial denominado TPP (Trans Pacific Partnership), uno de los acuerdos comerciales más importantes que ha firmado Canadá, donde algunos de los países firmantes, como Australia y Nueva Zelanda, se opusieron públicamente a la entrada de Canadá a dicho tratado comercial, a menos que desmantelara todas las entidades de supply management mencionadas arriba.
Varios autores canadienses han coincidido en decir que estos sistemas de control de la oferta no son más que subsidios gubernamentales a la producción, cuyas enormes consecuencias a nivel internacional castigan a Canadá en sus tratos comerciales con muchos países, particularmente con EE. UU., país que presiona frecuentemente al Gobierno federal canadiense para que desmantele, de una vez por todas, tales entidades y permita el libre juego de factores económicos (Clemens \& Crowley (Eds.), 2012).

Finalmente, hay voces dentro del Parlamento canadiense que acusan al Gobierno federal de miopía respecto a los objetivos económicos, aduciendo que, si bien Canadá ha logrado mantener estas entidades de control del suministro agropecuario dentro de sus esquemas de comercio con EE. UU. y la UE, está perdiendo mucho ante la imposibilidad de participar en otros esquemas de comercio con economías de mayor crecimiento y diversificación.

Para los exportadores españoles, es importante señalar que el control sobre el volumen de las importaciones que estas entidades de supply management realizan supone una grave cortapisa para la expansión fluida de las exportaciones españolas de productos alimenticios como los productos lácteos. Trátese de quesos o de otros derivados lácteos como helados o yogures, el establecimiento de cuotas de importación plantea una problemática insuperable para muchos exportadores internacionales.

En los próximos apartados se abordará con mayor detalle esta problemática, abundando en el modus operandi de la adjudicación de cuotas de importación y su impacto en el mercado de productos lácteos.

Baste decir que incluso en los círculos allegados al Gobierno federal existen dudas $\square$ 
sobre el futuro de las entidades de control del suministro agropecuario. Son conscientes de que las presiones de la globalización hacen insostenible el mantenimiento de tales entidades. Sin embargo, también son conscientes de que un abrupto desmantelamiento traería consecuencias irreversibles para la economía agrícola canadiense, no solo por la invasión de productos baratos americanos, sino porque alrededor de estas entidades de control del suministro se ha creado una red de intereses entre productores, importadores y distribuidores que son muy difíciles de romper.

Para culminar, hay que señalar que la emergencia sanitaria de la COVID-19, que afecta a Canadá como al resto de sus principales socios comerciales y a casi todo el mundo, además de haber ralentizado el comercio y el ritmo económico de todo el mundo, modifica y, por lo menos, cuestiona las principales formas de distribución internacional. La pandemia trae un olor a nuevo proteccionismo, basado en la inseguridad sanitaria que puede alterar la fluidez del comercio internacional. Esto lleva agua fresca al molino de los defensores del sistema de control del suministro agrícola en Canadá.

\section{Trabas a la importación de productos lácteos y sus derivados impuestas por los sistemas de control a la oferta en Canadá: el ejemplo de los quesos artesanales}

Como quedó establecido en el apartado anterior, los sistemas de control de la oferta de productos frescos en Canadá, como los lácteos y otros, determinan tanto el volumen de la producción total de lácteos como los precios de referencia que se pagan a los granjeros canadienses. Evidentemente, si el Gobierno federal, a través de la Canadian Dairy Comission, controla la producción nacional de quesos, el Departamento de Asuntos Exteriores (Global Affairs) canadiense deberá establecer un sistema de control de los volúmenes de lácteos que se importan, al objeto de lograr el control absoluto del mercado canadiense.

Considerando que el uso de términos es importante, hay que definir lo que en Canadá se denomina comúnmente «cuotas de importación de queso». Para efectos de este artículo, entendemos como "contingentes de importación de queso» aquellos volúmenes de producto negociados por Canadá en tratados internacionales con otros países suministradores.

Por otra parte, entendemos como «cuotas de importación de queso» determinados volúmenes de queso, establecidos de manera anual, que el Gobierno federal distribuye por medio de una licencia de importación a los operadores canadienses, que van prioritariamente a los fabricantes canadienses de queso y también a distribuidores o detallistas.

\subsection{Contingentes de importación negociados con la UE}

Durante décadas, las fronteras canadienses se mantuvieron cerradas a la importación de quesos por los motivos de control sobre la producción de lácteos analizados en epígrafes anteriores. Las únicas autorizaciones posibles se hacían de manera casuística, por medio de licencias de importación, al objeto de cumplir con las obligaciones comerciales impuestas a Canadá por la Organización Mundial de Comercio.

A partir de 2015, y durante el largo proceso de negociación y ratificación del Acuerdo Económico y Comercial Global entre Canadá y la Unión Europea (CETA), existía la esperanza $\triangleright$ 
entre la comunidad exportadora española de que dicho acuerdo viniera a liberalizar el sistema de importación de productos lácteos en Canadá, particularmente los quesos artesanales. Sin embargo, muy pronto se hizo evidente que los negociadores canadienses defendían a ultranza mantener un sistema de contingentes de importación consistente con los sistemas internos de control de la producción y, por ende, un severo control de las importaciones de queso.

Sin embargo, y debido al interés canadiense por tener un acceso al mercado europeo de productos lácteos, durante la negociación del CETA la parte canadiense ofreció un incremento sobre el volumen anteriormente aceptado para importación de quesos europeos que puede considerarse sustancial. Por añadidura, cabe mencionar que el acuerdo CETA finalmente protege a varias denominaciones de queso importantes, incluidas dos indicaciones geográficas de origen español: Queso Manchego, el más popular en el mercado, y Mahón-Menorca.

Este sistema de contingentes de importación de quesos que Canadá ha negociado con la UE está basado en volumen y no en valor, y se establece en dos partes: una parte histórica fijada en la década de los setenta y otra establecida con base en el acuerdo CETA firmado en el año 2017.

En el marco del acuerdo CETA entre Canadá y la Unión Europea y sus Estados miembros, Canadá convino proporcionar acceso adicional a los miembros de la UE específicamente para sus quesos. La cantidad de acceso al contingente arancelario del queso en el marco del CETA es de hasta 16.000.000 kilogramos para todos los tipos de quesos destinados al consumo y 1.700 .000 kilogramos para todos los quesos que serán utilizados con fines industriales o de procesado.
Estos contingentes de queso que ha negociado Canadá con la UE se distribuyen gradualmente a lo largo de cinco años, en seis plazos desde 2017 y hasta 2022, para una gestión más eficiente del impacto en la industria canadiense del queso. Por supuesto, una vez que el volumen de estos contingentes se agota, la única manera de importar quesos de un determinado país de la UE es a través del pago de onerosos aranceles, que pueden llegar al 245,5 ad valorem y no menos de 3,53 CAD $/ \mathrm{kg}$ en el caso del queso artesano y de 5,33 CAD/kg para el queso azul.

El contingente de importación ofrecido a los países de la UE se considera como una cantidad total, no existiendo un reparto específico de la cantidad total entre los países europeos. Se desconocen los procesos internos que han dado lugar a las asignaciones, pero, en contra de lo dispuesto en el anexo 2-B del CETA sobre administración de cuotas, estas no parecen estar contribuyendo a la llegada de nuevos países entrantes. En virtud de lo anterior, el Gobierno federal de Canadá ha iniciado un proceso de revisión de los procedimientos de adjudicación de cuotas de importación de todos los contingentes arancelarios, incluyendo el contingente de quesos CETA.

Cuando se analiza el ranking mundial de las importaciones de queso durante los años de vigencia del CETA, se observa que, según el origen de las importaciones de queso, nueve de los primeros quince países de origen pertenecen a la UE. Destaca la posición de Francia e Italia como principales suministradores de queso a Canadá, seguidos del vecino estadounidense. Durante 2020, España ocupó el puesto n. -10 del ranking. Lo anterior habla de que uno de los beneficios tangibles del CETA es la posibilidad de suministrar una mayor cantidad de quesos europeos en el mercado canadiense. 


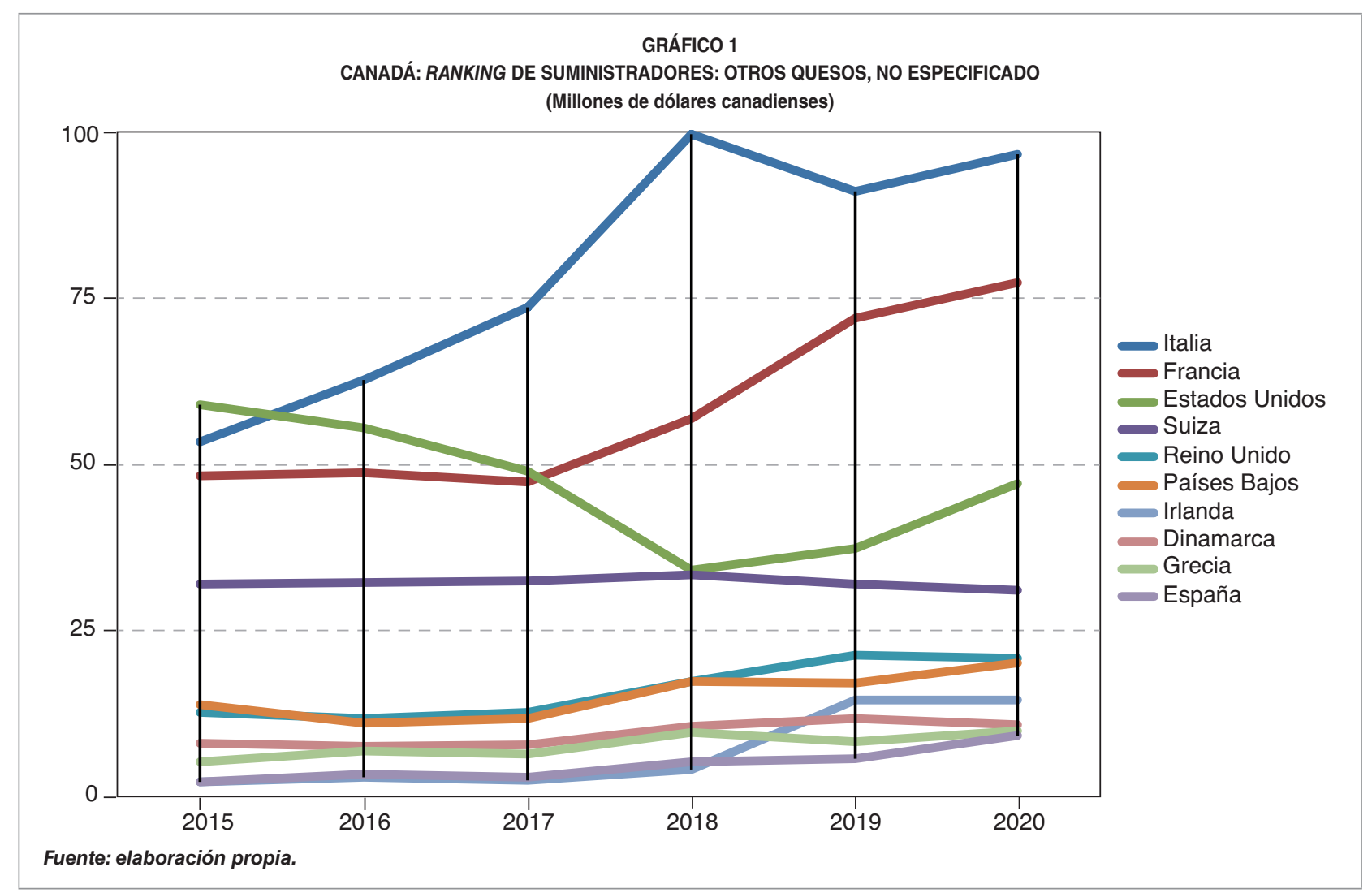

Hay que tener en cuenta también que, siendo dicho contingente en principio equitativo para todos los países europeos, el papel de España como suministrador de queso a Canadá es relativamente discreto y aún dista de las posiciones hegemónicas que juegan otros países europeos, como Francia o Italia, principales proveedores de queso a Canadá.

\subsection{Distribución de las cuotas de importación entre los operadores canadienses}

Desde hace décadas el Gobierno canadiense entiende la adquisición de quesos extranjeros como un complemento a la oferta de quesos que se producen a nivel nacional. Por tanto, la distribución de licencias de importación o cuotas TQR (por su nombre en inglés) se maneja como una prebenda para los fabricantes de queso que la solicitan y para los que se denominan importadores históricos (antiguos productores o distribuidores y retailers).

A nivel nacional, y de acuerdo con el directorio de empresas productoras de queso que facilita Canadian Dairy Information Centre, en Canadá hay censadas 198 empresas que elaboran distintos tipos y variedades de queso. De esta cantidad total, 112 están establecidas en la provincia de Quebec, lo que ofrece una idea de su potencial productivo en el contexto nacional, justificando también su aportación a la producción global de Canadá. En estos términos, la provincia de Quebec produjo, en 2019, un total de 249.000 toneladas de queso, alcanzando casi el $50 \%$ del total nacional (515.000 toneladas).

Con la entrada en vigor del CETA en 2017 se modifica el sistema de asignación de $\square$ 


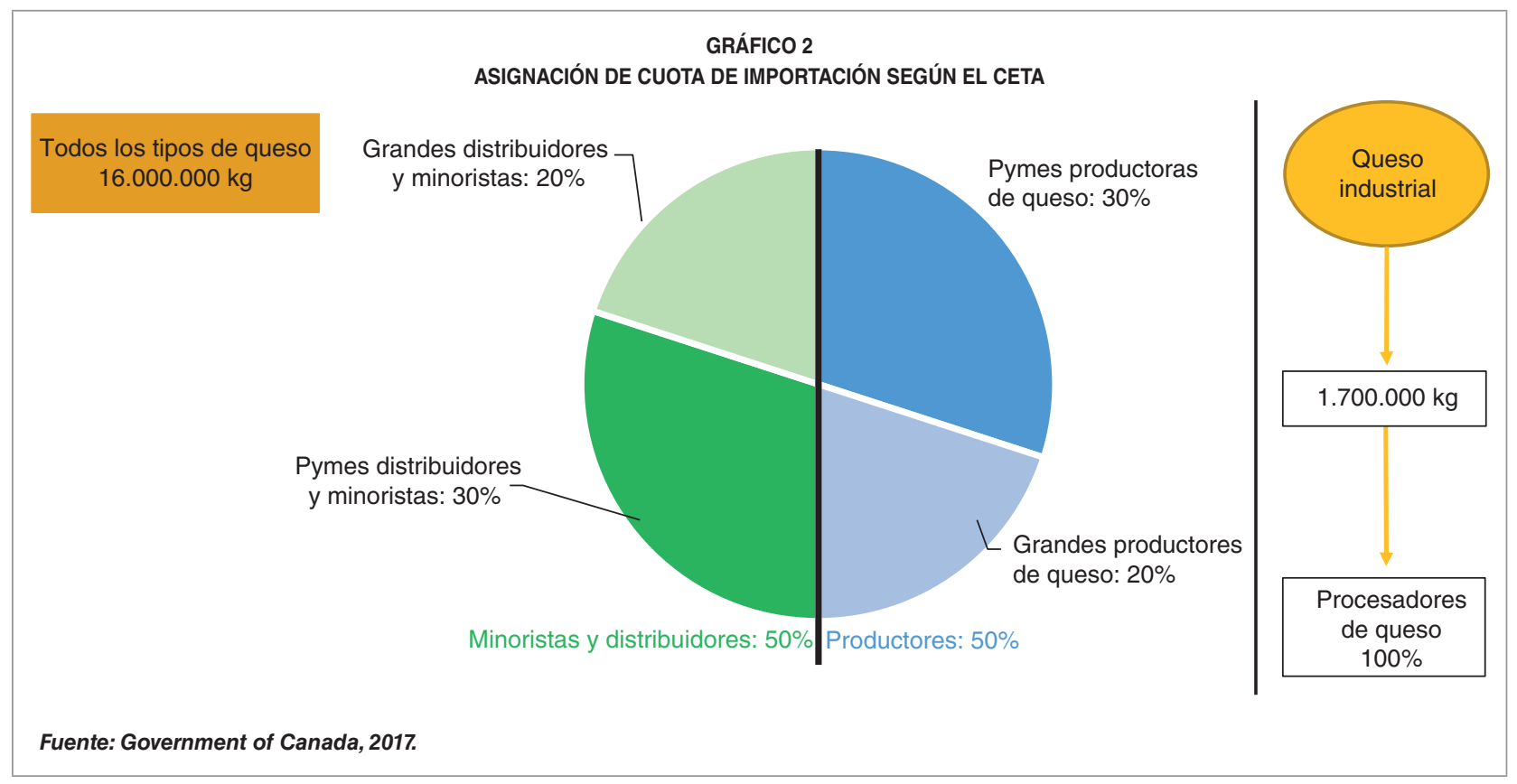

cuotas para importadores canadienses, ofreciendo un cupo de dichas asignaciones para lo que se denomina nuevos entrantes. La asignación a los distintos grupos de empresas que hace el Gobierno de Canadá para el queso procedente de la UE (en virtud del acuerdo CETA) se explica en el Gráfico 2, en el que se aprecia el reparto de dicha cuota a lo largo de la cadena de valor del queso, incluyendo fabricantes, distribuidores y minoristas de queso, y reservando partes de la cuota específicamente para las pequeñas y medianas empresas.

Para el año 2021, la idea es que por lo menos el $30 \%$ de la cuota deba asignarse cada año a nuevos entrantes (solicitantes que no dispongan de cuota OMC, o sea el sistema anterior al CETA) y a partir de 2022, por lo menos el $10 \%$ de la cuota se asignará a nuevos entrantes.

El sistema de asignación interna de cuotas para los importadores es donde debería hacerse más estable. La modesta ampliación de los receptores de licencias de importación de quesos, añeja demanda de los actores de la distribución alimentaria en Canadá, no consigue liberalizar y dar transparencia al mercado de quesos artesanales en Canadá.

Este intrincado sistema de asignación de cuotas de importación, desgraciadamente, se presta a la concentración de mayores volúmenes en muy pocas manos y sigue siendo una cortapisa importante para que nuevos suministradores y nuevos importadores canadienses logren hacerse un hueco en el mercado.

Los importadores canadienses se siguen quejando, y con razón, de que el sistema de asignación de cuotas responde a mecanismos poco transparentes en cuanto a las cantidades de importación asignadas, pero sobre todo la queja fundamental es la incertidumbre de no saber con qué volumen de cuota de importación pueden contar de un año a otro, ya que las asignaciones son erráticas y no responden a ninguna coherencia, por lo menos a los ojos de los propios operadores canadienses.

La anterior situación imposibilita una planificación a largo plazo, incrementa exponencialmente el riesgo de cualquier inversión conducente al desarrollo de marketing para apoyar $\triangleright$ 
algún tipo de queso y, por supuesto, este sistema encarece sobremanera el precio de estos productos, que son percibidos por el consumidor medio canadiense como demasiado caros para su presupuesto familiar.

En conclusión, es de celebrar el incremento de los contingentes establecidos para los suministradores europeos y el reconocimiento y protección de algunas denominaciones de origen de algunos quesos europeos que el CETA ofrece. Sin embargo, el mecanismo utilizado por el Gobierno federal canadiense para asignar las cuotas de importación bajo licencia a los importadores canadienses sigue siendo un cuello de botella casi infranqueable, que se está convirtiendo, de hecho, en una práctica proteccionista si consideramos lesiva para la expansión del consumo de queso al consumidor canadiense, ya que, al mismo tiempo que encarece el producto, limita la diversidad de variedades disponibles para el consumidor canadiense.

\section{Las limitaciones de los regímenes provinciales de importación de bebidas alcohólicas en Canadá}

El sector de las bebidas alcohólicas en Canadá está lleno de complejidades y restricciones. Aunque son similares en muchos aspectos, cada una de las diez provincias y tres territorios tiene su propio sistema, regido por sus respectivos Gobiernos provinciales, comúnmente designados «jurisdicciones de bebidas alcohólicas» 0 liquor boards. También tienen que cumplir directrices federales. Por lo tanto, se puede decir que el mercado canadiense no es un solo mercado, sino trece mercados distintos, cada uno de los cuales tiene su propio conjunto de limitaciones en lo que respecta a todo producto alcohólico, sea importado o doméstico.
Dependiendo de la jurisdicción, el sector se divide en una o más secciones, cada una de ellas dirigida por su propio órgano gubernamental y con una función diferente. Una de las ramas se ocupa del aspecto legislativo, por ejemplo, dónde se pueden vender bebidas alcohólicas tanto en on-trade como el off-trade, así como de las licencias pertinentes; y la otra se ocupa más específicamente de la importación, el almacenamiento, la distribución, la comercialización y la venta, más comúnmente conocida como liquor boards.

Hay cuatro provincias que representan el $86,97 \%$ del mercado total de ventas de bebidas alcohólicas en Canadá: Ontario, Quebec, Columbia Británica y Alberta, y, como era de esperar, cada una tiene un reparto porcentual muy diferente entre las distintas categorías de bebidas. Por ejemplo, Quebec (23,25\%) tiene una cuota de ventas más alta que Alberta $(10,70 \%)$, y en Ontario la proporción de ventas $(37,90 \%)$ es mayor que en Columbia Británica $(15,12 \%)$. El resto de las ventas totales de Canadá se distribuyen entre las demás provincias y territorios, que también representan proporciones muy diferentes para las distintas bebidas alcohólicas (cerveza, vino, alcohol y bebidas preparadas) que se venden en cada una de ellas. Para comprender las limitaciones del sistema canadiense, dada la estructura que presentan los distintos niveles de gobierno, es necesario hacer un breve resumen de su funcionamiento básico.

En general, y usando como ejemplo las dos provincias más grandes, Quebec y Ontario, la mayoría de las ventas se realizan a través de la red de venta que es propiedad y está gestionada por cada uno de los Gobiernos provinciales: la SAQ tiene 409 tiendas y la LCBO tiene 669 tiendas. Fundamentalmente, estos dos monopolios provinciales (liquor boards) tienen $\triangleright$ 
el mismo sistema en lo que respecta a como listan los productos en sus tiendas. Se trata de un sistema casi totalmente de licitación (tender process) en el que se publica una lista de convocatoria de productos que incluye todas las especificaciones (país de origen, región, coste de venta al público, premios y diversos plazos de pago). Las presentaciones se envían electrónicamente en nombre del productor por su agente, que tiene que estar oficialmente reconocido por la autoridad provincial del mercado de su interés. Si el producto es aceptado, se procede a la degustación y prueba, y se examinan los planes de comercialización.

Aunque hay varias especificaciones que deben acordarse para que un producto se incluya en la oferta del liquor board, las condiciones de pago, el apoyo a la comercialización en términos de dólares y el acuerdo para alcanzar las cuotas de ventas establecidas son tres factores clave para que un producto sea aprobado y aceptado. Cuanto más largas sean las condiciones de pago y mayor sea el apoyo de marketing, mayores serán las posibilidades de que el producto se incluya en la oferta. $Y$ en cuanto a las cuotas, si no se cumplen, el producto será retirado de la lista y/o se cobrarán sanciones monetarias al proveedor.

Aunque no es obligatorio, casi todos los productores tienen un agente, ya que son ellos los que conocen y entienden los entresijos del mercado y dialogan con los liquor boards en nombre del productor. La figura del agente es muy importante, ya que deberá conocer ampliamente el mercado y todas las idiosincrasias del mismo, encargándose de realizar las tareas pertinentes en él y ante los liquors boards.

Continuando con las provincias de Ontario y Quebec, existe otro canal llamado consignación por el que los agentes traen el producto que quieren vender a los clientes de HORECA o al consumidor particular. Estos productos son casi totalmente exclusivos de este canal. En este caso, los monopolios provinciales realizan también la importación del vino y reciben sus fondos de los costes del almacenamiento.

En Columbia Británica existe un sistema híbrido en el que las ventas se realizan tanto a través del sistema de tiendas del liquor board (435 tiendas oficiales) como del sector de tiendas privadas (1.415 establecimientos). Por el momento, existe una moratoria para la aprobación de licencias nuevas a más tiendas del sector privado en Columbia Británica. El sistema de la Junta de Licores de esta provincia es muy similar al de Quebec y Ontario, y, aunque existen algunas diferencias operativas, la red de tiendas funciona básicamente de la misma manera. En cuanto a la red de tiendas privadas, se venden productos que, en su mayoría, no están disponibles en las tiendas oficiales. Naturalmente, esto proporciona una mayor variedad al consumidor, pero también da a los agentes y a los minoristas un poco más de flexibilidad en lo que respecta a los márgenes. La última de las grandes provincias es Alberta, donde el sistema de tiendas es cien por cien privado a través de tiendas independientes. Sin embargo, para que los fondos sigan entrando en las arcas provinciales, el Gobierno mantiene el control de la importación y el almacenamiento de las bebidas alcohólicas. Los sistemas provinciales y sus diferentes ramas se pueden consultar en la Tabla 1.

Hay que destacar que el mercado en Canadá está empezando a abrirse en su segmento minorista. Incluso en Ontario, el vino y la cerveza importados están llegando poco a poco a las estanterías de los supermercados. Cabe señalar que hasta 450 tiendas de comestibles están autorizadas para vender cerveza y sidra, de las cuales alrededor de 150 también venden vino. 


\section{LA LETRA PEQUEÑA DE LA EXPORTACIÓN DE ALIMENTOS A CANADÁ}

TABLA 1

CANALES DE DISTRIBUCIÓN POR PROVINCIAS

\begin{tabular}{|c|c|c|c|}
\hline Provincia & Liquor board & Canal de venta & Doing business \\
\hline Alberta & https://aglc.ca/ & https://liquorconnect.com/ & $\begin{array}{l}\text { https://aglc.ca/liquor/ } \\
\text { warehouse-and-distribution }\end{array}$ \\
\hline Columbia Británica & $\begin{array}{l}\text { https://www2.gov.bc.ca/gov/content/ } \\
\text { employment-business/business/ } \\
\text { liquor-regulation-licensing }\end{array}$ & http://www.bcldb.com/ & $\begin{array}{l}\text { http://www.bcldb.com/doing-business-ldb/ } \\
\text { information-bc-liquor-manufacturers }\end{array}$ \\
\hline Ontario & https://www.agco.ca/ & https://www.lcbo.com/content/lcbo/en.html & $\begin{array}{l}\text { https://www.doingbusinesswithlcbo.com/ } \\
\text { content/dbwl/en/basepage/home.html }\end{array}$ \\
\hline Manitoba & https://lgcamb.ca/ & https://www.liquormarts.ca/ & https://www.mbllpartners.ca/ \\
\hline Nuevo Brunswick & \multicolumn{3}{|c|}{ https://www.anbl.com/ } \\
\hline Terranova y Labrador & \multicolumn{3}{|c|}{ https://nlliquor.com/ } \\
\hline Territorios del Noroeste & \multicolumn{3}{|c|}{ https://www.fin.gov.nt.ca/en/services?combine=\&field_service_category_tid=401\&field_program_type_tid=All } \\
\hline Nueva Escocia & \multicolumn{2}{|c|}{ https://www.mynslc.com/ } & https://www.mynslc.com/trademynslc \\
\hline Nunavut & \multicolumn{2}{|c|}{ https://www.nulc.ca/ } & $\begin{array}{l}\text { https://www.gov.nu.ca/finance/information/ } \\
\text { governing-documents }\end{array}$ \\
\hline Isla del Príncipe Eduardo & \multicolumn{3}{|c|}{ https://liquorpei.com/ } \\
\hline Saskatchewan & \multicolumn{3}{|c|}{ https://www.slga.com/ } \\
\hline Quebec & https://www.racj.gouv.qc.ca/en/home.html & https://www.saq.com/en/ & https://www.saq.com/en/suppliers \\
\hline Yukón & https://alcohollaws.ca/yukon/ & https://yukon.ca/en/liquor-products-prices & https://yukon.ca/en/doing-business \\
\hline
\end{tabular}

La existencia de los liquor boards introduce limitaciones o trabas que dificultan el acceso al mercado de las bebidas alcohólicas importadas.

En primer lugar, es necesario mencionar que las bodegas canadienses pueden vender sus productos desde las bodegas mismas y también algunas tienen tiendas privadas de distribución al público de sus productos. El vino que se vende en estas tiendas, caso que sea el vendido también en el liquor board, debe mantener el mismo precio. Si se trata de un vino no listado en el mismo, la bodega canadiense puede pedir el precio que estime oportuno, siempre que lo haga sin bajar por debajo del floor price establecido por el monopolio provincial.

El liquor board «lista» marcas de vino, de manera que esos vinos listados son los que están disponibles pare el público en las tiendas. Es, por tanto, el que decide qué productos importar y ofertar al público a través de su cadena de tiendas, y cuándo retirarlos del mercado en base a un sistema de cuota de venta establecido por dicho organismo. Para ello, estudia las ofertas que le hacen las bodegas nacionales y extranjeras (estas a través de agentes) siguiendo un procedimiento que se podría considerar estándar, aunque con ciertas diferencias en cada provincia. Además, de manera muy excepcional contacta directamente con los productores cuando está interesado en algún producto en particular y, del mismo modo, también de manera muy excepcional puede tomar en consideración peticiones privadas para la adquisición de productos que no se encuentran entre los que ofrecen en las tiendas, siempre que se respeten unos mínimos de compra.

Añadido a esto está el sistema de fijación de categorías en las tiendas por parte del monopolio, en muchos casos por países o grupos de países, que afecta al posicionamiento de las bodegas y su ubicación en las tiendas. Cabe destacar a este respecto el trato de favor concedido a los productos locales respecto del otorgado a su competencia extranjera. 
En resumen, Canadá no es un único mercado, sino varios mercados diferentes. Al tratar con liquor baords surgen limitaciones mayores a las «habituales» de cara al acceso al mercado y se pueden generar problemas de discrecionalidad, subjetividad, y falta de transparencia. Los liquor boards no siempre están bien gestionados y se producen muchos problemas de ruptura de existencias y mala gestión que provocan descensos en las ventas de las marcas afectadas sin que la bodega pueda hacer nada al respecto. $Y$ cabe destacar la discriminación frente a la producción doméstica, sobre todo en Ontario y Columbia Británica, las provincias de mayor producción de vino; los caldos locales tienen mejor posicionamiento en estanterías, mayores oportunidades de promoción otorgadas por los liquor boards, tiendas propias de venta al público además de las de aquellos y, en algunos casos, márgenes más favorables.

\section{Régimen de inspección sanitaria para productos cárnicos en Canadá}

Durante la época victoriana, en Inglaterra empieza a legislarse la inspección de productos cárnicos. Siendo Canadá miembro de la Commonwealth, en lo que todavía se denominaba Upper Canada, ya existían algunas formas de regulación sanitaria del tratamiento de los productos cárnicos. Incluso desde principios del siglo xIx existían reglas de curado, envase y transporte de cárnicos, aunque su aplicación en el territorio canadiense era laxa y muy dispar según el territorio o provincia que lo ejerciera.

Sin embargo, no es hasta la publicación en 1907 de lo que se denominó Acta Canadiense de Carnes y Productos Enlatados, legislada por el Parlamento federal, cuando se establece una normativa específica que cubre todas las actividades de sacrificio, corte y futuro proceso de cárnicos en el país. Asimismo nace simultáneamente un servicio de vigilancia e inspección que vela por la óptima sanidad de estos productos.

Las primeras disposiciones de esta legislación aluden a la necesidad de crear un registro de plantas existentes en todas las provincias y la obligación para los particulares de obtener una licencia sanitaria para sus instalaciones, previa inspección y superación de las pruebas sanitarias establecidas.

La licencia además servía para conocer el origen del comercio de cárnicos entre las provincias y era indispensable para vender allende las fronteras canadienses. Sin embargo, los carniceros locales de Ontario que solo distribuían carne a su clientela urbana estaban exentos de conseguir la denominada licencia sanitaria. Desde 1920, los centros de sacrificio se ubican cerca de las estaciones de ferrocarril para que los granjeros puedan enviar sus animales, estableciéndose un rudimentario servicio de clasificación y registro sanitario.

Durante los años cincuenta, y con el advenimiento de alimentos producidos de manera industrial, particularmente aquellos denominados ready to eat que utilizaban grandes cantidades de estabilizadores, se dota a la legislación genérica «Food And Drugs Act» de una sección de alimentos cárnicos procesados y otros embutidos.

Para los años sesenta, tanto el registro como los sistemas de inspección se han sofisticado enormemente con la adopción, mediante legislación del Parlamento canadiense, de la denominada "The Meat Inspection Act», que entra en vigor en 1963. Dicha acta, además de establecer regulaciones muy precisas sobre la $D$ 
producción de distintos tipos de cárnicos, fija las bases de lo que actualmente se denomina «Normas de Higiene Canadienses para la Producción de Cárnicos».

Finalmente, y con motivo de la creación de la Canadian Food Inspection Agency (CFIA) en 1997, estas reformas continuas en la legislación sanitaria canadiense nos indican un intento de puesta al día sanitario, al efecto de que las exportaciones de cárnicos canadienses tengan mejores posibilidades en los mercados internacionales y particularmente en el creciente mercado norteamericano.

Asimismo, la legislación se orienta a contener las importaciones de productos peligrosos para la salud del hato canadiense. Estas medidas cobran fuerza en la legislación sanitaria de Canadá a partir de los brotes de fiebre aftosa experimentados en Europa entre los años 1991 y 2005, que afectaron particularmente a Reino Unido y Rusia, y, en menor medida, a Bulgaria y Albania.

Además, se presentan otros brotes de enfermedades, como la encefalopatía espongiforme bovina (EEB) o de las «vacas locas», que afectó incluso a España a principios del siglo xxI, y que arrasó con los hatos de Reino Unido, país que tenía un trato comercial agrícola muy intenso con Canadá.

Otra enfermedad sobre la cual Canadá mantiene una vigilancia permanente es la denominada fiebre porcina africana (ASF), que actualmente se concentra en países asiáticos y africanos, pero que ha tenido brotes también en países europeos.

Lo anterior es importante para entender el desarrollo del mercado canadiense de embutidos cárnicos porcinos europeos, como los jamones españoles (Serrano e Ibérico) y el prosciutto italiano. Según el Gobierno canadiense, el impacto de un brote de ASF en Canadá afectaría a
103.000 empleos directos, un valor de venta cercano a los 23.000 millones de dólares canadienses e impactaría en las exportaciones canadienses de productos porcinos con una baja del $20 \%$, siendo Canadá el tercer país en el ranking de exportación mundial, con casi 1,2 millones de toneladas exportadas en 2017.

Habida cuenta de lo anterior, los procedimientos de importación de productos cárnicos porcinos son extremamente rigurosos, lo cual se puede considerar como una barrera no arancelaria muy difícil de superar para cualquier exportador europeo, máxime cuando la fiebre porcina africana ha sido desterrada de los países como España, Francia e Italia desde hace décadas.

A modo de ejemplo del alto grado de sobrerregulación, en la siguiente página ofrecemos el documento denominado Condiciones de Importación que publica la CFIA (mediante el sistema interactivo AIRS) y que lista cada requerimiento indispensable para la importación de un solo producto cárnico procesado y proveniente de España (jamón).

\section{Regulación sobre envases de alimentos en Canadá}

En el caso de los envases de alimentos, la legislación canadiense busca objetivos muy concretos a fin de defender la seguridad alimentaria del consumidor canadiense. Por tanto, su reglamentación mediante la denominada Food \& Drugs Act tiene como objetivo detectar en los envases cualquier sustancia venenosa o que pueda causar daño a la salud humana, determinar si el estado del contenido es apto para el consumo humano y no tiene muestras de suciedad, putrefacción o si ha sido adulterado. Finalmente, la legislación correspondiente $\triangleright$ 


\section{RECUADRO 1}

\section{REQUISITOS DE REGISTROY DOCUMENTACIÓN PARA LA EXPORTACIÓN DE JAMÓN}

\section{Recommendations to CBSA (Canada Border Services Agency)/Documentation and Registration Requirements:}

Refer to CFIA-NISC (must be accompanied by the following documents|registrations):

1. Official Meat Inspection Certificate

2. Canadian Meat Inspection Establishment (other than US shipments)

3. Transport Carrier

4. Foreign Processing Establishment

5. Product packaging type

6. Number of packages

7. Total Weight/Volume
8. Export establishment

9. Shipping marks

10. Seal number

11. Slaughter establishment

12. Total weight unit of measure

13. Container number

14. Safe Food for Canadians License

Or Refer to CFIA-NISC (must be accompanied by the following documents/registrations):

1. Official Meat Inspection Certificate

2. Canadian Meat Inspection Establishment (other than US shipments)

3. Transport Carrier

4. Foreign Processing Establishment

5. Product packaging type

6. Number of packages

7. Total Weight/Volume

\section{Importer / Broker Instructions}

Documentation instructions

Under the Safe Food for Canadians Act (SFCA) and Safe Food for Canadians Regulations (SFCR), many food businesses require a license:

- To carry out activities with respect to food.

- To import food into Canada.

Businesses must obtain their SFC license before presenting their shipment at the border. They will not be able to obtain an SFC license at the border.

When applying for a license:

- Ensure that it covers importing food as well as the food commodity or commodities you are importing.

When submitting your import information:

- Provide your SFC license number, along with all other pertinent information about your shipment.

A Safe Food for Canadians license number is to be entered as part of an import declaration in the applicable AIRS Registration Type (\#893 - Safe Food for Canadians).

vela por que la fabricación y el estado de los envases reúnan todas las condiciones sanitarias del caso.

Asimismo, la Food \& Drugs Act (F\&D Act) establece que el Departamento Federal de Salud (Health Products and Food Branch o HPFB) y la CFIA serán las entidades que tengan las competencias de establecimiento de políticas conducentes a un sistema de inspección sanitaria integral en todo el territorio canadiense.

Una de las normas jurídicas de la legislación establece que se prohíbe la venta de cualquier producto alimenticio que contenga cualquier sustancia nociva para la salud, y que será el vendedor final el responsable único de cualquier infracción. 


\section{LA LETRA PEQUEÑA DE LA EXPORTACIÓN DE ALIMENTOS A CANADÁ}

\section{RECUADRO 2 \\ REQUISITOS DE IMPORTACIÓN E INSTRUCCIONES ADICIONALES PARA LA IMPORTACIÓN DE JAMÓN}

\section{Conditions of import}

Importers are responsible for ensuring that the food they import for sale into Canada comply with the requirements of all applicable Canadian legislation including Safe Food for Canadians Act and Regulations, and the Food and Drugs Act and Regulations.

\section{Official meat inspection certificate}

For samples less than $100 \mathrm{~kg}$ and personal importation less than $20 \mathrm{~kg}$ imported from designated countries, a zoo sanitary certificate is acceptable in lieu of the official meat.

\section{Designated countries}

Designated means a country must be free of the following diseases depending on which species the meat is derived from (fresh or processed meat): Bovine, Ovine and Caprine: Foot-and-Mouth Disease.

For Bovine Spongiform Encephalopathy (BSE), Canada accepts the World Organization for Animal Health (OIE) classification for country BSE risk categorization. Refer to the BSE Import Policy for Bovine Animals and Their Products and By-Products within the additional references section for list of countries by category.

Porcine: Foot-and-Mouth Disease, Swine Vesicular Disease, Classical Swine Fever and African Swine Fever.

Avian: Newcastle and Highly Pathogenic Avian Influenza.

May be subject to inspection at first point of arrival. Must proceed to an inland facility designated for inspection of imported meat products.

May be subject to inspection at first point of arrival. Must proceed to an inland establishment where it is stored and handled in its imported condition by a license holder. For full import conditions for various meat products please refer to country specific Conditions of importation of meat products.

\section{Additional instructions}

Guidelines to follow when submitting a faxed package to NISC:

- Do not use company fax cover page.

- Use the 'Request for Documentation Review' form (CFIA/ACIA 5272) as the first document of a fax submission.

- Ensure the new version of the 'Request for Documentation Review' form (CFIA/ACIA 5272) is used.

- Ensure all information on the 'Request for Documentation Review' form (CFIA/ACIA 5272) is typed.

- Do not provide a bar code and/or a label transaction code on the form CFIA/ACIA 5272.

- Ensure to key in the transaction number on the form CFIA/ACIA 5272.

- Do not affix any labels or stickers to the form CFIA/ACIA 5272.

- Should a single fax contain multiple requests ensure the 'Request for Documentation Review' form CFIA/ACIA 5272 separates each request.

\section{Wood packaging material (for all origins except continental United States)}

Brokers/ Importers must attest to one of the following with respect to the packaging material being used to ship the product:

- "Wood packaging contained with shipment displays the ISPM No. 15 compliant stamp."

- Or Wood packaging material is accompanied by a Phytosanitary Certificate ie.

- Or Wood packaging material is made of manufactured wood which is exempted from ISPM No. 15."

- Or (when it applies) "No wood packaging contained with shipment."

\section{Fully marked}

- These shipments can be admitted into Canada and to a registered establishment if they arrive in a container without a seal.

- These shipments can be released into general commerce in Canada in their imported state and be admitted to a registered establishment without the seals.

\section{Instructions for CBSA}

D-19 - Edible Meat and Meat Products.

Included in This Section:

- Any edible meat or poultry and any food product containing meat or poultry in any amount.

- Any edible meat and meat products in-bond, in transit and Canadian edible meat and meat products exported and returned to Canada. 
Según la F\&D Act, el envase alimenticio se define como cualquier utensilio usado como continente de comida (parcial o total). Tan genérica definición permite al HPFB una interpretación que incluye y sujeta bajo su inspección sanitaria todo artículo, equipo o transporte que entre en contacto con el envase de comida, no solo desde el proceso de fabricación, sino también en su distribución, incluida en los establecimientos de venta. Por supuesto, todo lo que es requerido al fabricante nacional puede ser requerido a los suministradores extranjeros.

Asimismo, como ejemplo, el Departamento de Salud prohíbe la utilización de ciertos materiales de empaque o embalaje fabricados con cloruro de polivinilo cuya fórmula contenga octylestaño.

Evidentemente, ninguno de estos productos de envase y embalaje va a entrar en contacto con la comida, sea en lata o en envases de plástico, pero según la interpretación del HPFB, son sujetos de regulación e inspección por la CFIA. Lo mismo se aplica para los containers, transportes o módulos de exhibición en supermercados.

Aunque en Canadá, a diferencia de la legislación estadounidense del FDA, no es necesario el prerregistro para la importación de alimentos de consumo humano, el Departamento de Salud canadiense ofrece la posibilidad de emitir una opinión sobre los envases o embalajes que contienen a los alimentos antes de su distribución en el mercado. Por tanto, y a solicitud voluntaria del fabricante o importador de comida procesada, el HPFB puede emitir lo que se denomina como "Letter of No Objection», o «NOLO», siempre mediante una «voluntaria» solicitud del interesado y donde se hace una revisión sanitaria de todos los envases y embalajes que según su interpretación podrían entrar en contacto con la comida.
Por supuesto que estas NOLO no constituyen una aprobación oficial del Departamento de Salud para los materiales de envase y embalaje, tampoco eximen al solicitante de las penas que devienen de una inspección. Sin embargo, pueden facilitar la expedición de mercancías cuya importación se realiza de manera frecuente. Incluso algunos fabricantes obtienen las NOLO por motivos de prestigio mercadológico.

Hay que mencionar, asimismo, que, aunque el Departamento de Salud o la CFIA no publican un listado de materiales de envase y embalaje que estén, a priori, para uso de la industria alimenticia, en el portal del Departamento de Salud existe un listado de aquellos polímeros que han recibido NOLO positiva.

Por último, es importante recalcar que, en términos generales, los materiales de envase y embalaje aceptados por la FDA de EE. UU. pueden ser aceptables en Canadá. Sin menoscabo de lo anterior, el sometimiento a la legislación canadiense resulta indispensable. EL HPFB realizará una revisión científica independiente, utilizando sus propios métodos para estimar el grado de exposición de los alimentos con relacion a los materiales de envase y embalaje.

\section{Conclusión}

A la luz de las múltiples restricciones que afectan y limitan la importación en Canadá de varios productos claves de la gastronomía española, como los quesos artesanales, los vinos de mesa, los productos cárnicos de cerdo como los jamones, tanto Ibérico como Serrano, y de aquellos productos envasados en origen, como el aceite oliva o los mariscos finos, consideramos de gran utilidad continuar con el asesoramiento al exportador español sobre $\square$ 
los vericuetos administrativos que hemos llamado «la letra pequeña de la importación en Canadá».

Sin embargo, consideramos que el paso trascendental para incrementar tanto cuantitativa como cualitativamente el flujo comercial entre España y Canadá es reiterar, ante las instancias negociadoras correspondientes, que las partes contratantes del CETA revisen y, en su caso, modifiquen aquellos esquemas que se consideren sobrerregulados, al objeto de posibilitar una mayor fluidez y transparencia en las estructuras y en los procesos administrativos de importación de productos alimenticios, en ambos lados del Atlántico.

\section{Bibliografía}

Barter, H. C. (2014). Slaughterhouse Rules: declining abattoirs and the politics of food safety regulation in Ontario. University of Toronto. Canada. https://tspace.library.utoronto.ca/bitstream/ 1807/68573/1/Barter_Hillary_C_201411_MA_ thesis.pdf

Canadian Dairy Commission. (2020). Dairy Products Marketing Regulations. Frequently Asked Questions-Regulations. Government of Canada. https://cdc-ccl.ca/index.php/en/about-the-canadian-dairy-commission/acts-and-regulations/ frequently-asked-questions-regulations-edit/

Canadian Dairy Commision. (2021). Roles and responsibilities. Responsibilities of the federal departments and agencies. Government of Canada. https://cdc-ccl.ca/index.php/en/dairyindustry/federal-and-provincial-responsibilities/

Canada Revenue Agency. (2012). Amendment to the Importation of Intoxicating Liquors Act. Government of Canada. https://www.canada.ca/ en/revenue-agency/services/forms-publications/ publications/edn31/amendment-importation-intoxicating-liquors-act.html
Clemens, J., \& Crowley, B. L. (Eds.). (2012). Milking the System. How Agricultural Suply Management Impedes Trade Opportunities and Egregiously Transfers Income. Hungry for change series. Macdonald-Laurier Institute. https://www. macdonaldlaurier.ca/files/pdf/Supply-Management-Essays-June-2012.pdf

Global Affairs Canada. (2017). Canada's new tariff rate quotas for cheese imports from the European Union. Government of Canada. https:// www.canada.ca/en/global-affairs/news/2017/08/ backgrounder_-_canadasnewtariffratequotasforcheeseimportsfromthe.html

Global Affairs Canada. (2018). Dairy - CETA Cheese Tariff Rate Quota (TRQ) (Items 141 to 157 on the Import Control List). Government of Canada. https://www.international.gc.ca/controls-controles/prod/agri/dairy-laitiers/notices-avis/909. aspx?lang=eng

Heminthavong, K. (2015). Canada's Supply Management System. Library of Parliament.

International Chesee Council of Canada. (s.f.). The Global Affairs Canada (GAC) Tariff Rate Quotas (TRQ) Review. http://www.internationalcheesecouncil.ca/news/the-global-affairs-canadagac-tariff-rate-quotas-trq-review/

LCBO, Liquor Control Board of Ontario. (2021). LCBO Pricing Structure: Price Markup Example. https:// hellolcbo.com/app/answers/detail/a_id/1251/ / Icbo-pricing-structure\%3A-price-markup-example

Misko, G. G. (26 de Febrero de 2021). The Use of Printing Inks for Food Packaging in the United States. FoodSafety Magazine. https://www. food-safety.com/articles/7001-the-use-ofprinting-inks-for-food-packaging-in-the-unitedstates

Parreño Parreño, J. (2020). Estudio de mercado. El mercado del vino en Canadá 2020. Icex España Exportación e Inversiones. https://www.icex.es/ icex/es/navegacion-principal/todos-nuestros-servicios/informacion-de-mercados/paises/ navegacion-principal/el-mercado/estudios-informes/estudio-mercado-vino-canada-2020doc2020866291.html?idPais=CA 
Statistics Canada. (2021a). Sales of alcoholic beverages by liquor authorities and other retail outlets, by type of outlet. https://www150.statcan.gc. $\mathrm{ca} / \mathrm{t} 1 / \mathrm{tb} 11 / \mathrm{en} / \mathrm{tv}$.action? pid $=1010001301 \&$ pickMembers $\% 5 B 0 \% 5 \mathrm{D}=1.11$ \&pickMembers\%$5 \mathrm{~B} 1 \% 5 \mathrm{D}=2.3 \& \mathrm{cubeTimeFrame.start-}$ Year $=2019+\% 2 F+2020 \&$ cubeTimeFrame. endYear $=2019+\% 2 F+2020 \&$ referencePeriods $=20190101 \% 2 \mathrm{C} 20190101$

Statistics Canada. (2021b). Value of sales of alcoholic beverages of liquor authorities and other retail outlets, by beverage type (x 1,000). https:// www150.statcan.gc.ca/t1/tbl1/en/tv.action? pid $=1010001101 \&$ pickMembers $\% 5$ B 0 \%
$5 \mathrm{D}=1.1 \& \mathrm{pickM}$ e m be r s $\% 5$ B $1 \%$ $5 \mathrm{D}=2.1 \&$ cubeTimeFrame. startYear $=2019+\%$ $2 \mathrm{~F}+2020$ \& $\mathrm{c} \mathrm{u} \mathrm{b}$ e T i m e F r a m e. endYear $=2019+\% 2 F+2020 \&$ referencePeriods $=20190101 \% 2 \mathrm{C} 2019011$

Taric Wisetech Global Group. (s.f.). Contingente arancelario. https://www.taric.es/productos-yservicios/utilidades/glosario/contingentearancelario/

Veeman, M. M. (2011). Agricultural Marketing Board. The Canadian Encyclopedia. https://www.thecanadianencyclopedia.ca/en/article/ agricultural-marketing-board 